\title{
Especie nueva de Justicia (Acanthaceae) del bosque tropical caducifolio de la costa de Oaxaca, México
}

\author{
A new species of Justicia (Acanthaceae) from the tropical deciduous forest of the Oaxacan \\ coast, Mexico
}

Salvador Acosta-Castellanos

Centro Interdisciplinario de Investigación para el Desarrollo Integral Regional (CIIDIR) Unidad Oaxaca, Instituto Politécnico Nacional. Dirección actual: Laboratorio de Palinología, Departamento de Botánica, Escuela Nacional de Ciencias Biológicas, I.P.N., Apartado postal 17-564, 11410, México D. F., México.

Correspondencia: salvacst@yahoo.com.mx

Resumen. Se describe Justicia salma-margaritae del bosque tropical caducifolio de la costa de Oaxaca, México. La nueva especie se agrupa en Justicia sección Sarotheca y se caracteriza por la combinación de sus inflorescencias espigadas compuestas, cáliz desigualmente 5-lobado, tecas de las anteras sin apéndices, polen 2-colporado con 4 filas de ínsulas en el área tremal, cápsulas pubescentes y semillas tuberculadas. Sin embargo, se puede diferenciar claramente de las especies previamente descritas por la peculiar pubescencia glandular que presentan las brácteas, bractéolas y segmentos del cáliz.

Palabras clave. Oaxaca, México, Justicia salma-margaritae, Acanthaceae.

Abstract. Justicia salma-margaritae is described from the tropical deciduous forest of the Oaxacan coast, Mexico. It is placed in Justicia sect. Sarotheca on account of a combination of morphological characters: compound spicate inflorescences, unequally 5-lobed calyces, unappendaged anther thecae, 2-colporate pollen with 4 rows of insulae in the trema area, pubescent capsules and tuberculate seeds. However, it may be clearly differentiated from the previously described species of that section by the peculiar glandular pubescence of bracts, bracteoles and calyces.

Key words. Oaxaca, Mexico, Justicia salma-margaritae, Acanthaceae.

\section{Introducción}

El género Justicia L. (Acanthaceae), de distribución pantropical, es uno de los más numerosos de la familia, con más de 600 especies reconocidas (Graham, 1988). En México se conocen cerca de 78 especies (Acosta y Daniel, 1993; Daniel, 1999) pero con la exploración botánica de diversas regiones de México, se han descrito especies no reconocidas previamente de éste y otros géneros de la familia, en su mayor parte de las zonas tropicales (Daniel, 1999; Daniel, 2002).

Durante la revisión de la familia Acanthaceae del estado de Oaxaca, principalmente de la región de la costa, se encontraron ejemplares de una especie poco colectada. Posteriormente se colectó más y mejor material y después de su análisis se concluyó que se trata de una nueva especie.

Recibido: 27 abril 2006; aceptado: 18 octubre 2006

\section{Descripción}

Justicia salma-margaritae Acosta. sp. nov. TIPO: México. Oaxaca: Distrito Pochutla, municipio de San Miguel del Puerto, camino de la Laguna de las Garzas a la playa, $4 \mathrm{~km}$ al ESE de Barra de Copalita. elev. 20 m, N 15 47' 49", O 96 01' 25", 11 enero 2001, S. Acosta 3208 (Holotipo: OAX; isotipos: MEXU, ENCB, CAS). Figura 1.

Suffrutex ad 2 m altus, ramosus, caules erecti, glabri. Folia opposita, petiolata vel subsessilia; limbus lanceolatus vel ovato-lanceolatus, glabratus, margine integro, apice acuto vel apiculato, base in petiolum decurrenti vel obtusa. Spicae dichasiales pedunculatae. Flores sessiles. Bracteae, bracteolae et calices laciniis glanduloso- pubescentibus cum pilis bruneis peltatis glandulosis et pilis simplicibus intermixtis. Calyx 5-partitus, laciniis inaequalibus, linearisubulatis. Corolla purpurea bilabiata, extus pubescens, intus glabrata (tubi parte angustata dense pubescenti excepta). Stamina 2, vix exserta; antherae biloculares, 


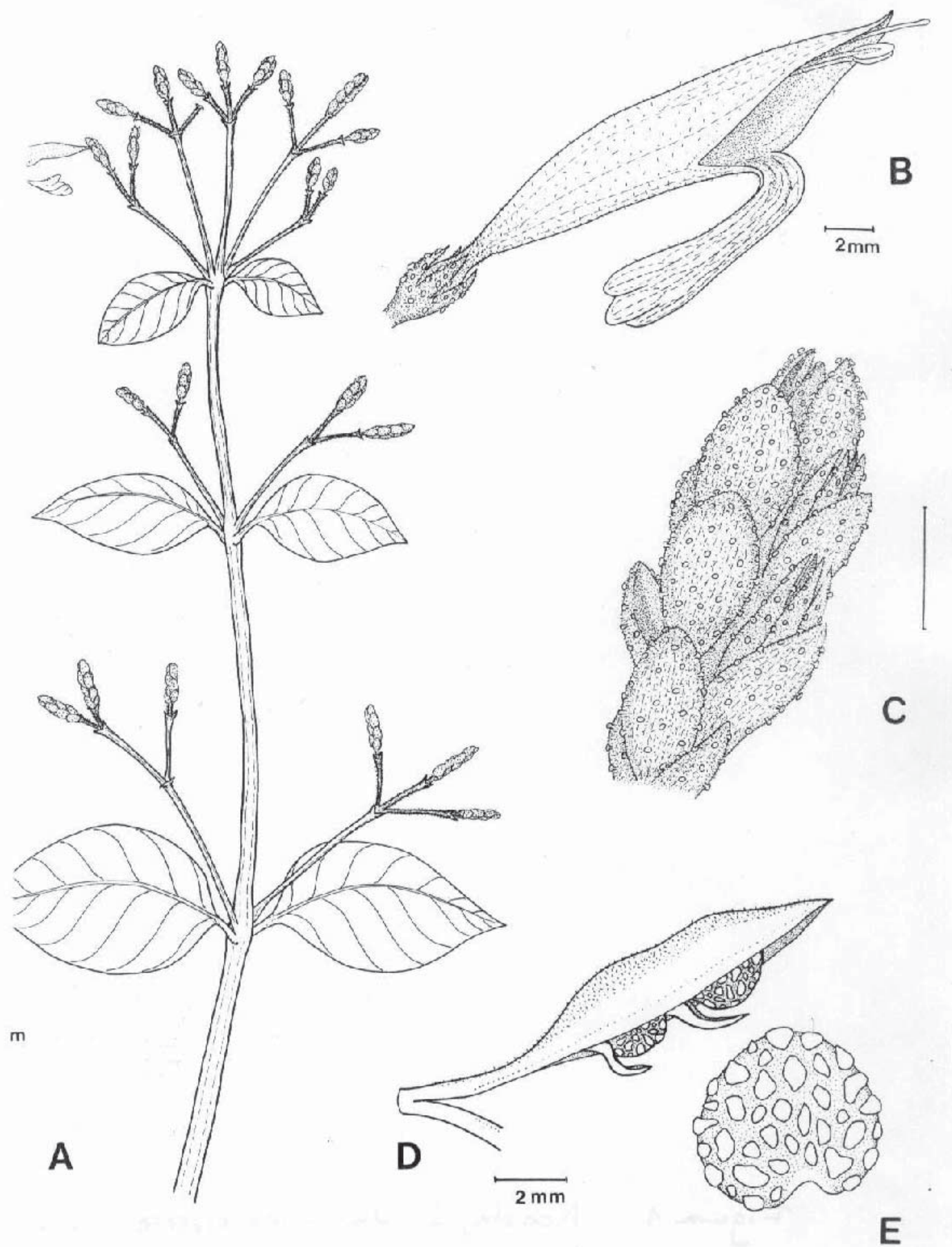

Figura 1. Justicia salma-margaritae. A, rama terminal; B, corola y cáliz; C, detalle de una espiga; D, cápsula (parte); E. semilla. 
thecis inaequaliter insertis, subsagittatis, non calcaratis. Pollinis granula bicolporata, prolata, 2 seriebus insularum in quoque latere pororum. Capsula stipitata, glandulosopubescens. Semina 2-4, cordiformia, tuberculata.

Subarbusto de $80-200 \mathrm{~cm}$ de altura, ramificado desde la base; ramas erguidas, glabrescentes, circulares en sección transversal; hojas opuestas, subsésiles a pecioladas, pecíolos de 2-23 $\mathrm{mm}$ de longitud, glabros; láminas hasta de $110 \mathrm{~mm}$ de longitud y $62 \mathrm{~mm}$ de ancho, 1.5-2 veces más largas que anchas, lanceoladas a ovado lanceoladas, con el ápice agudo a apiculado, decurrentes a redondeadas, con el margen entero, las hojas del mismo par subiguales, haz y envés glabros, con cistolitos evidentes, las nervaduras laterales más o menos alternas, de 3-5 (6) pares. Inflorescencias terminales y axilares, espigas pedunculadas dispuestas en forma de dicasio, pedúnculos glabros de 18-40 mm de largo; las espigas de 5-19 $\mathrm{mm}$ de longitud, pediceladas, pedicelos de 2-6.8 mm de longitud, pedicelos densamente puberulentos, con tricomas muy cortos, simples; raquis con pubescencia similar; brácteas basales (de la inflorescencia) largamente triangulares, agudas, verdosas, glabras, de 1.9-2.6 mm de longitud y 1.0-1.2 mm de ancho; brácteas y bractéolas externamente glandular pubescentes con tricomas glandulares peltados de color café dispuestos principalmente en la mitad superior de 0.12-0.14 mm de longitud y 0.10-0.12 mm de diámetro y tricomas simples entremezclados de cerca de $0.024 \mathrm{~mm}$ de largo, internamente glabras o con algunos tricomas simples esparcidos, con cistolitos lineares evidentes; brácteas elípticas a ovado-lanceoladas de 2-3.4 mm de longitud y 1.3-2.0 mm de ancho, obtusas; bractéolas largamente triangulares, verdosas, agudas, de 2.6-3.6 mm de longitud y de 0.72-1.0 mm de ancho. Flores sésiles 1 por nodo; segmentos del cáliz 5, desiguales, uno de ellos muy reducido, agudo, menor de $1 \mathrm{~mm}$ de longitud (0.86-0.9 mm), los otros cuatro linear-subulados, de 2.2-3.1 mm de longitud y 0.6-0.8 $\mathrm{mm}$ de ancho, la parte interna ligeramente pubescente, con tricomas simples y la externa con tricomas glandulares, peltados, de color café y tricomas muy cortos entremezclados, ambos de dimensiones similares a los presentes en las brácteas y bractéolas. Corola bilabiada, purpúrea (fucsia, rosaíndigo), superficie externa pubescente, con tricomas eglandulares, simples, superficie interna glabra excepto en la estrechez del tubo donde es densamente pubescente, de 23-30 mm de longitud; tubo de la corola de $2 \mathrm{~mm}$ de diámetro en la base, estrechándose hasta $1 \mathrm{~mm}$ antes de abrirse gradualmente en la garganta; labio superior triangular, entero, subagudo, de 7.0-10.0 mm de longitud y $8.0 \mathrm{~mm}$ de ancho, con un par de pliegues alargados en la parte interna cerca del ápice, de aproximadamente 0.5 $\mathrm{mm}$ de ancho; el labio inferior ligeramente más largo que el superior, de 8-13 mm de largo, reflexo, trilobulado, los lóbulos subiguales, elíptico-alargados, redondeados, de 2.6-4.8 mm de longitud y de 2-3.5 mm de ancho. Estambres 2 , casi de la misma longitud que el labio superior o ligeramente exertos, insertos cerca del ápice del tubo de la corola (a $1.5 \mathrm{~mm}$ por debajo de la comisura del labio, a una distancia de 15-18 mm desde la base), filamentos glabros, de 7.3-8.3 mm de longitud, anteras subsagitadas, biloculadas, con las tecas desigualmente insertas, una de ellas reducida, múticas, de 1.8-2.3 mm de largo. Polen 2colporado, prolato, con 2 series de ínsulas a cada lado de los poros, la superficie interapertural reticulada (Fig. 2). Disco cupular, de color verdoso, de $1 \mathrm{~mm}$ de diámetro y $0.9 \mathrm{~mm}$ de alto. Ovario glabro, con 2 óvulos por lóculo; estilo glabro, filiforme, hasta de $28 \mathrm{~mm}$ de largo; estigma subcapitado. Cápsula estipitada, de (7.5) 10-13.5 mm de longitud y de 2.8-3.4 mm ancho, estípite de (4) 5.2-7.5 mm de longitud, apiculada, glandular pubescente, con tricomas
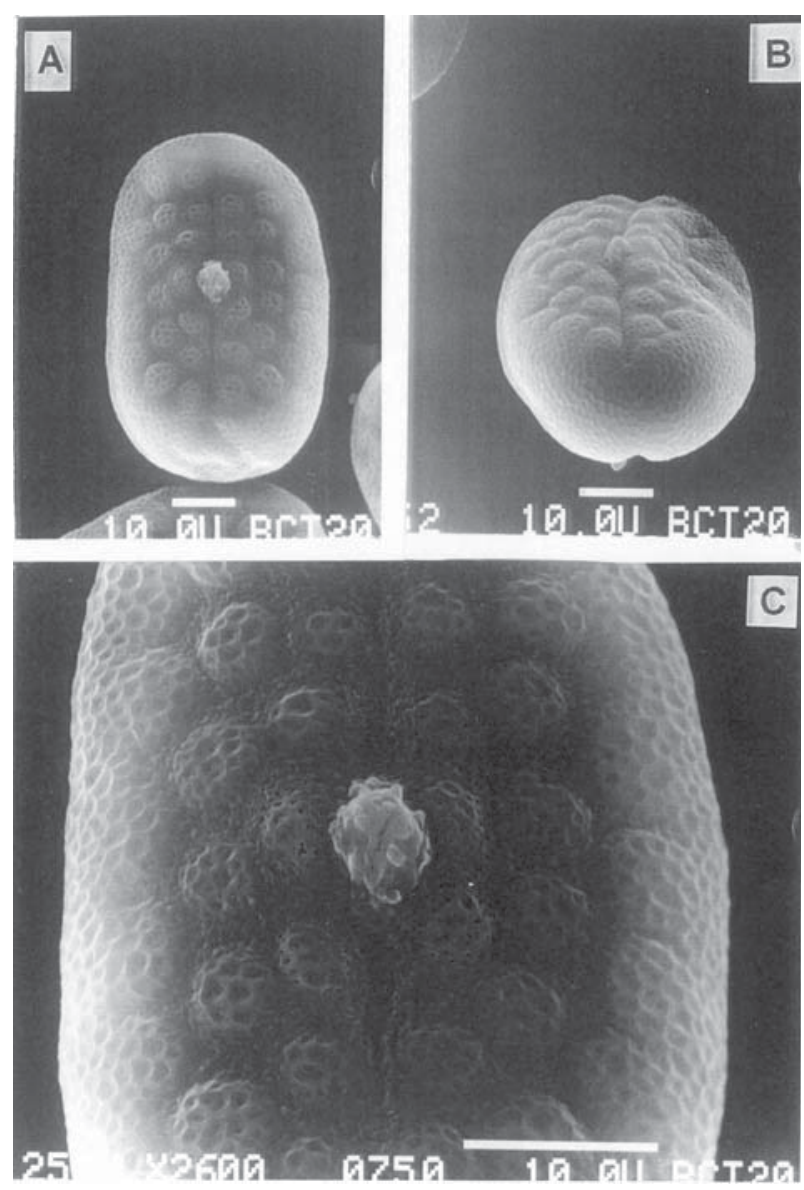

Figura 2. Microfotografías de polen de Justicia salma-margaritae (E. Martínez 33142) en el microscopio electrónico de barrido (MEB). A, vista ecuatorial; B, vista de la zona interapertural en uno de los polos; $\mathrm{C}$, detalle del colporo y las ínsulas (vista ecuatorial). 
cortos entremezclados. Semillas 2-4, a veces sólo una por aborto de las demás, cordiformes, comprimidas, superficie tuberculada, de 2-2.5 mm de diámetro.

Se encontró en floración de octubre a enero y en fructificación en diciembre y enero.

\section{Resumen taxonómico}

Material adicional estudiado. Oaxaca: Distrito Tehuantepec: municipio Tehuantepec, Santa Cruz Bamba (Mamba en la etiqueta), $10 \mathrm{~km}$ al SO de Morro Mazatán, carr. Salina Cruz- Pochutla, N 1601', O 95 25', dic. 23, 1988, C. Martínez 2211 (MEXU); municipio Santiago Astata, $2 \mathrm{~km} \mathrm{~N}$ de Barra de la Cruz, alt. $75 \mathrm{~m}$, N 1550' 50”, O 95 58' 46”, 22 noviembre 1999, E. Martínez 33142, 33158 (MEXU); municipio Santiago Astata, camino hacia Zimatán (La Mareña), 4 km al SO de Barra de la Cruz, alt. 120 m, N 15 49' 26', O 95 58' 03", 10 enero 1999, M. Elorsa 1447 (OAX); municipio Santiago Astata, playa Barra de la Cruz, N $15^{\circ} 49^{\prime} 2^{\prime \prime}, \mathrm{O}$ 95 58' 8", 16 noviembre 1999, M. Elorsa 2481 (MEXU, SERO); municipio Santiago Astata, $2.2 \mathrm{~km}$ NE de Barra de la Cruz, arroyo Agua Grande, alt. $45 \mathrm{~m}, \mathrm{~N} 15^{\circ} 51^{\prime}$ 12",O 95 57' 05”, 22 diciembre 1999, M. Elorsa 2573 (MEXU, SERO); municipio Santiago Astata, 4 km NE de Barra de la Cruz, ladera arriba del puente del Ciruelo, alt. $70 \mathrm{~m}, \mathrm{~N} 15^{\circ} 52^{\prime}$, O 95 57' 15', 6 diciembre 1999, J. F. Castrejón 1103 (MEXU, SERO); municipio Santiago Astata, $4 \mathrm{~km} \mathrm{~N}$ Barra de la Cruz, sobre el cerro, alt. 20 m, 13 diciembre 2001, M. Elorsa 5520 (MEXU, SERO); municipio San Pedro Huamelula, San Isidro Chacalapa, $3.7 \mathrm{~km} \mathrm{~N}$ de la desviación en la carretera costera, alt. 190 $\mathrm{m}, \mathrm{N} 15^{\circ} 54^{\prime}, \mathrm{O} 95^{\circ} 55^{\prime} 33^{\prime \prime}, 4$ diciembre 1999, S. Salas 2577 (MEXU, SERO); municipio Santiago Astata, vereda vieja a Zimatán, $2 \mathrm{~km}$ al NE de Barra de la Cruz, alt. $20 \mathrm{~m}$, N 15 50' 36.6”, O 95 58' 47', 13 noviembre 2003, M. Elorsa 7593 (SERO).

Distribución y hábitat. Se ha encontrado en el bosque tropical caducifolio (selva baja y mediana caducifolia) de la vertiente del océano Pacífico en los distritos de Pochutla y Tehuantepec del estado de Oaxaca, México. Crece desde el nivel del mar hasta los $190 \mathrm{~m}$ de altitud.

\section{Comentarios taxonómicos}

Justicia salma-margaritae se puede ubicar en la sección Sarotheca (Nees) Benth., por sus inflorescencias en forma de espigas dicasiales, de una flor por nodo, cáliz 5-partido con un segmento reducido y semillas con superficie tuberculada, aunque difiere de dicha sección porque el eje de la inflorescencia es solamente pubescente y no glandular, las tecas de las anteras no están superpuestas, ni oblicuas, no poseen apéndices y el polen es más bien tipo
7 (Graham, 1988) y no tipo 6. Las especies de la sección Sarotheca provienen del Nuevo Mundo y se encuentran principalmente en Centro y Sudamérica.

Acosta y Daniel (1993) mencionaron que la clasificación infragenérica de Justicia propuesta por Graham (1988) resultó problemática en el caso de la circunscripción de la sección Dianthera subsección Saglorithys, con relación a la ubicación de J. santelisiana Acosta et Daniel, otra especie del S de Oaxaca. Por otro lado, Daniel (1999, 2002) menciona que numerosas especies neotropicales de Justicia no pueden clasificarse en ninguno de los taxa infragenéricos reconocidos. Se requiere una revisión de la clasificación infragenérica de Justicia, la cual sólo será posible con el mayor conocimiento de todas sus especies.

La especie se dedica a la memoria de una maravillosa joven estudiante, Salma Margarita Portillo Macías (19802001), a quien quisiéramos mantener por siempre cerca los que extrañamos su compañía.

\section{Agradecimientos}

Al Dr. Fernando Chiang, del Instituto de Biología, Universidad Nacional Autónoma de México, quien amablemente revisó el manuscrito y corrigió la diagnosis en latín. A Daniel Acosta Aguilar de la Facultad de Artes Plásticas de la Universidad Veracruzana, por realizar la ilustración de la especie. A la Dra. Silvia Antuna Vizarro, de la Facultad de Medicina de la Universidad Nacional Autónoma de México y la M. en C. Leonor Quiroz García, de la Escuela Nacional de Ciencias Biológicas, quienes tomaron las microfotografías al MEB. A los encargados de los Herbarios ENCB, MEXU, OAX, SERO y XAL, por facilitar la consulta de las colecciones. Al Centro Interdisciplinario de Investigación para el Desarrollo Integral Regional (CIIDIR) Unidad Oaxaca, por su apoyo durante las consultas a los herbarios mencionados, y a los tres revisores anónimos que hicieron valiosas sugerencias para mejorar el artículo.

\section{Literatura citada}

Acosta, S. y T. F. Daniel, 1993. A new species of Justicia (Acanthaceae) from southern Mexico. Kew Bulletin 48: 119-123.

Daniel, T. F. 1999. Taxonomic and distributional notes on Neotropical Justicia (Acanthaceae). Proceedings of the California Academy of Sciences 51: 483-492.

Daniel, T. F. 2002. New and reconsidered Mexican Acanthaceae IX. Justicia. Proceedings of the California Academy of Sciences 53: 37-49.

Graham, V. 1988. Delimitation and infra-generic classification of Justicia (Acanthaceae). Kew Bulletin 43: 551-624. 